\title{
Bilateral Nephrectomy for Adult Polycystic Kidney Disease Does Not Affect the Graft Function of Transplant Patients and Does Not Result in Sensitisation
}

\author{
Maria Irene Bellini (D), ${ }^{1}$ Sotiris Charalmpidis, ${ }^{1}$ Paul Brookes, ${ }^{2}$ Peter Hill, ${ }^{1}$ \\ Frank J. M. F. Dor, ${ }^{1,3}$ and Vassilios Papalois ${ }^{1,3}$ \\ ${ }^{1}$ Renal and Transplant Directorate, Hammersmith Hospital, Imperial College Healthcare NHS Trust, London, UK \\ ${ }^{2}$ Department of Pathology, Hammersmith Hospital, Imperial College Healthcare NHS Trust, London, UK \\ ${ }^{3}$ Department of Surgery and Cancer, Imperial College, London, UK
}

Correspondence should be addressed to Maria Irene Bellini; mariairene.bellini@nhs.net

Received 17 March 2019; Accepted 19 May 2019; Published 11 June 2019

Academic Editor: Deepak Nihalani

Copyright (C) 2019 Maria Irene Bellini et al. This is an open access article distributed under the Creative Commons Attribution License, which permits unrestricted use, distribution, and reproduction in any medium, provided the original work is properly cited.

Background. Native nephrectomy in Adult Polycystic Kidney Disease (ADPKD) patients is a major operation with controversy related to timing and indications. We present our single centre experience in transplanted patients and future candidates for transplantation. Methods. Retrospective analysis from an anonymised database of bilateral nephrectomies for ADPKD patients. Results were reported as median, range, and percentage. Differences between groups were tested using ANOVA and t-test. Surgery was performed between January 2012 and July 2018. Results. Thirty-three patients underwent bilateral native nephrectomy for APKD. 18 had a functioning kidney transplant (transplant group, 55\%) while 15 patients were on dialysis (dialysis group, 45\%) at the time of surgery; 8 patients of the latter group ( $24 \%$ of the whole cohort) were eventually transplanted. $53 \%$ were males, with median age of 55 years (27-71). Indications to surgery were the following: space (symptoms related to the size of the native kidneys or need to create space for transplantation) (59\%), recurrent cyst infection (36\%), haematuria (15\%), pain (24\%), and weight loss associated with cystic alteration on imaging (3\%). In the transplant group, postoperative kidney function was not affected; haemoglobin serum levels significantly dropped in the whole cohort: $121(82-150) \mathrm{g} / \mathrm{L}$, versus 108 (58-154) g/L ( $\mathrm{p}<0.001)$, with 14 patients being transfused perioperatively. Elevation of anti-HLA antibodies was noted in one female patient on dialysis, with no change in DSA levels and no rejection after transplant for all 26 transplanted patients. Median postoperative length of hospital stay was 9 days (6-71). One patient died (3\%) after six months. Median follow-up for the whole cohort was 282 days (13-1834). Histopathological examination revealed incidental renal neoplasms in five cases (15\%): 1 pTla papillary renal cell carcinoma and 4 papillary adenomas. Conclusions. Native nephrectomy for ADPKD could be safely performed in case of refractory symptoms, suspect of cancer or to create space for transplantation. It does not affect graft function or DSA status of transplanted patients or the prospect of transplantation of those on the waiting list.

\section{Introduction}

Autosomal dominant polycystic kidney disease (ADPKD) is the consequence of a heterozygous mutation in one of two genes: PKD1 on chromosome 16 [1], in $80-85 \%$ of cases, or PKD2 on chromosome 4 [2]. The mutation leads to dysfunction of the corresponding protein products, polycystin 1 and 2 , resulting in aberrant cellular signalling pathways with increased or disorganised cell growth and fluid secretion with fluid accumulation and cyst formation $[3,4]$.

Clinical manifestations in ADPKD patients include urine-concentration defect, haematuria $[3,5]$, cyst infection, urinary tract infection [6], loin or abdominal pain, abdominal fullness and discomfort, nephrolithiasis, and hypertension [7]. An association with other systemic manifestations is also frequent: polycystic liver disease, mitral 
valve prolapse or ventricular hypertrophy [8], intracranial aneurysm, abdominal aortic aneurysm [7], diverticular disease [5], and bronchiectasis [8]. For abdominal aortic aneurysm in particular, they tend to expand more rapidly in transplanted patients, and in the case of massively enlarged polycystic kidneys, the surgical access for a ruptured abdominal aortic aneurysm could be very challenging [9]; therefore variation in clinical symptoms has to be carefully assessed.

The clinical course is variable, suggesting that other genes and environmental factors may play a role [10]. Progression of ADPKD is ultimately defined by the onset of end stage renal failure; ADPKD is the commonest inherited kidney disease and is the fourth commonest cause of kidney failure worldwide $[11,12]$.

The timing of surgical intervention is controversial for patients with refractory symptoms, especially if they are not in renal replacement therapy. Native nephrectomy of ADPKD is generally performed for recurrent infection, haematuria, space, chronic pain, and tumour suspicion on imaging [1315]. Literature reports that approximately $20-30 \%$ of the whole ADPKD population require surgical intervention [3, $16,17]$, although there is an associated morbidity that might jeopardise the possibility for the patients to get transplanted, so routine pretransplant nephrectomy is no longer recommended.

The aim of this study is to review our single centre experience with native bilateral nephrectomy for ADPKD in patients who were waitlisted for, or previously underwent kidney transplant, focusing on timing of surgery, surgical outcomes, postnephrectomy kidney graft function, and sensitisation.

\section{Patients and Methods}

The study was designed as a single-centre retrospective cohort analysis. We identified the patients who have undergone native bilateral nephrectomy of their polycystic kidneys in the period between January 2012 and July 2018 from our prospectively maintained database. All the patients underwent native bilateral nephrectomy through an open midline laparotomy and the specimens were submitted for routine histological evaluation. The decision to proceed with surgery depended on the indications for surgery and the perceived benefits, and it was made following consensus of a multidisciplinary team of surgeons, nephrologists, and radiologists. Based on our protocol, for predialysis patients, we wait until they need dialysis and we perform the nephrectomies after they are established on dialysis for a period of 3 months.

The study was conducted in accordance with institutional ethics regulations; since it was a retrospective chart analysis, no informed consent was required. Demographic, clinical, and laboratory information was extracted in an anonymised way from charts and electronic records, including operation reports and pre- and postoperative clinic notes: indications for surgery, imaging, estimated Glomerular Filtration Rate (eGFR) according to the Modification of Diet in Renal Disease formula [18], Donor Specific Antibodies (DSA), and
anti-HLA (Human Leucocyte Antigens), haemoglobin levels before and after surgery, and pathology reports. Patients were divided according to their transplant status: having a functioning kidney transplant (transplant group) or being on dialysis (dialysis group) at the time of native bilateral nephrectomy. Surgical morbidity was categorised according to Clavien classification [19].

Results are reported as median, range, and percentage. Differences between groups were tested using ANOVA and t-test. A critical $p$ value of 0.05 was set for statistical significance. Statistical analyses were performed using SPSS v.20.0 (IBM SPSS Statistics for Windows, Version 20.0; IBM Corp, Armonk, NY).

The work has been reported in line with the STROCSS criteria [20].

\section{Results}

Thirty-three consecutive patients were identified during the study period. Of these, 18 had a functioning kidney transplant at the time of the native bilateral nephrectomy $(55 \%) /$ transplant group, while 15 patients were on dialysis at the time of the operation (45\%)/dialysis group; 8 of the patients on dialysis at the time of the operation were later transplanted ( $24 \%$ of the whole cohort). Immunosuppression therapy consisted of alemtuzumab induction and tacrolimus maintenance monotherapy, with early withdrawal of steroids in the first week after transplant. The median follow-up was 282 days (33-1834). One patient (3\%) died 6 months after bilateral nephrectomy. $55 \%$ of the whole cohort were males $(n=18)$, with median age 55 years (range 27-71), Table 1. Age and sex distributions were the same between the transplant and dialysis groups.

Indications for native nephrectomy were categorised into space (symptoms related to the size of the native polycystic kidneys or need to create space for transplantation) $(n=20$, $59 \%)$, recurrent cyst infection $(n=12,36 \%)$, haematuria $(n=5,15 \%)$, pain $(n=8,24 \%)$, and weight loss associated with cystic alteration on imaging $(n=1,3 \%)$, with haemorrhagic and complex cyst content. Indications for surgery did not differ statistically between the transplant and dialysis groups.

In the transplant group, median eGFR before and at day 1 after native bilateral nephrectomy did not differ significantly: 44 (29-73), versus $45(27-90) \mathrm{ml} / \mathrm{min} / 1.73 \mathrm{~m}^{2}$ (paired t-test, $\mathrm{p}=0.63)$, as also shown in Figure 1.

Figure 2 shows the significant post-native bilateral nephrectomy change in the haemoglobin serum levels for the whole cohort, 121 (82-150) pre- versus 107 (58-154) g/L postoperatively $(\mathrm{p}<0.001)$.

Fourteen patients were transfused perioperatively (Clavien grade II). Elevation of anti-HLA post- compared to prebilateral native nephrectomy was noted only in one female dialysis patient, with previous history of pregnancies. For all 26 transplanted patients (18 transplanted before and 8 transplanted after native bilateral nephrectomy), during the follow-up period of this study, DSA levels did not change and there was no rejection episode. 
TABLE 1: Results: the estimated Glomerular Filtration Rate (eGFR) is expressed in $\mathrm{ml} / \mathrm{min} / 1.73 \mathrm{~m}^{2}$ and the haemoglobin (Hb) in g/L.

\begin{tabular}{|c|c|c|c|}
\hline & & Total $(\%)$ & Median (Range) \\
\hline \multicolumn{2}{|l|}{ Male } & $18(53)$ & \\
\hline \multicolumn{2}{|c|}{ Age at operation (years) (both groups) } & & $55(27-71)$ \\
\hline \multicolumn{2}{|c|}{ Age at operation (years) (transplant group) } & & $54(27-68)$ \\
\hline \multicolumn{2}{|c|}{ Age at operation (years) (dialysis group) } & & $59(40-71)$ \\
\hline \multirow{5}{*}{ Indication } & Discomfort/space & $20(59)$ & \\
\hline & Infection & $12(36)$ & \\
\hline & Haematuria & $5(15)$ & \\
\hline & Pain & $8(24)$ & \\
\hline & Weight loss and cystic content modification & $1(3)$ & \\
\hline \multicolumn{2}{|c|}{ Transplant pre-native bilateral nephrectomy } & $18(53)$ & \\
\hline \multicolumn{2}{|c|}{ Transplant post-bilateral native nephrectomy } & $8(24)$ & \\
\hline \multicolumn{2}{|c|}{ eGFR pre-nephrectomy (18 patients-transplant group) } & & $44(29-73)$ \\
\hline \multicolumn{2}{|c|}{ eGFR day 1 post-nephrectomy (18 patients-transplant group) } & & $45(27-90)$ \\
\hline \multicolumn{2}{|c|}{ Hb pre-native bilateral nephrectomy } & & $121(82-150)$ \\
\hline \multicolumn{2}{|c|}{ Hb day 1 post-native bilateral nephrectomy } & & $107(58-154)$ \\
\hline \multicolumn{2}{|c|}{ Transfusion } & $14(42)$ & \\
\hline \multicolumn{2}{|c|}{ Anti-HLA or DSA level change } & $1(3)$ & \\
\hline \multicolumn{2}{|c|}{ Length of hospital stay (days) } & & $9(6-71)$ \\
\hline
\end{tabular}

DSA: Donor Specific Antibody. HLA: Human Leukocyte Antigen.

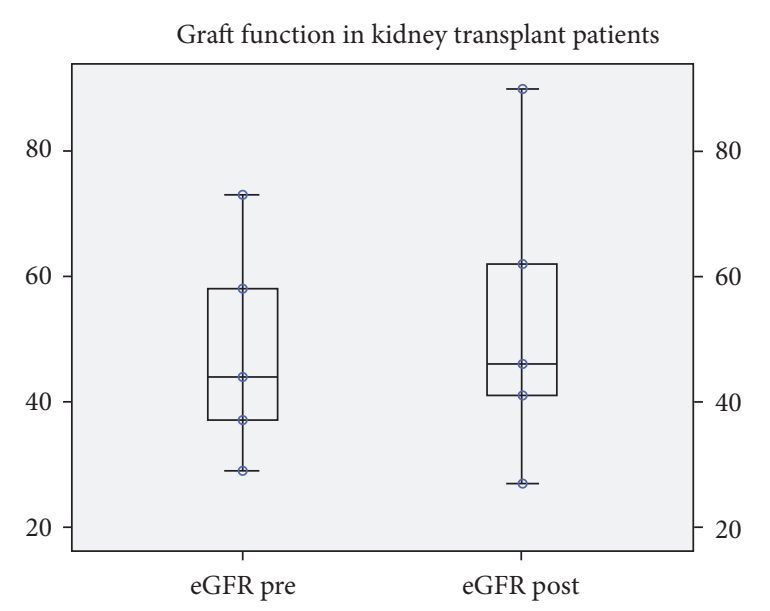

FIGURE 1: Median eGFR before nephrectomy and on the $1^{\text {st }}$ postnephrectomy day. No significant effect in kidney function was noted $(\mathrm{p}=0.63)$. eGFR is reported in $\mathrm{ml} / \mathrm{min} / 1.73 \mathrm{~m}^{2}$.

Median postoperative length of hospital stay (LOS) was 9 days (6-71): $56 \%$ up to 9 days, $75 \%$ up to 11 days, and $97 \%$ up to 24 days. One patient developed line sepsis, requiring in-hospital antibiotic treatment during dialysis, and was discharged on day 71 . There was no statistical difference between the transplant and the dialysis groups $(p=0.28)$. Two patients developed prolonged ileus that spontaneously resolved within 1 week of the operation (6\%) (Clavien grade I); two patients developed abdominal collections and were treated with intravenous antibiotics (Clavien grade II), plus interventional radiology drain insertion in one case (Clavien grade III). One patient developed refractory ascites due

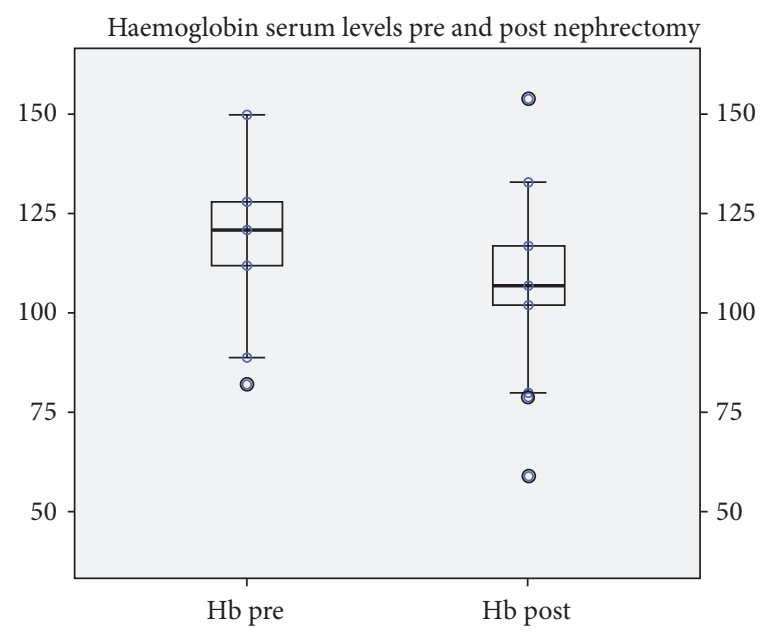

FIgURE 2: Haemoglobin levels $(\mathrm{Hb})$ before nephrectomy and on the $1^{\text {st }}$ postnephrectomy day, with lower levels postoperatively $(\mathrm{p}<0.001)$. Hb is reported in $\mathrm{g} / \mathrm{L}$.

to liver disease, finally leading to hepatorenal syndrome (Clavien grade IV); this patient was initially discharged but died 6 months after the operation. No patient underwent relaparotomy postoperatively.

The specimens demonstrated typical features of ADPKD in all cases, with replacement of kidney tissue by numerous cysts. Median cyst diameter was $7.5 \mathrm{~cm}$ (3.5-10), median kidney length $22 \mathrm{~cm}$ (7.5-38), median width $14 \mathrm{~cm}$ (6-19), median height $10 \mathrm{~cm}$ (3-14), and median weight $1631 \mathrm{~g} \mathrm{(566-}$ 5840). Figures 3(a) and 3(b) demonstrate massive polycystic kidneys leading to an indication for creating space in a patient 


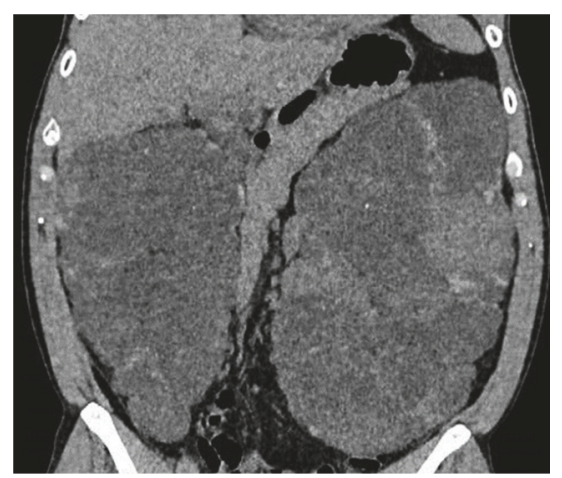

(a)

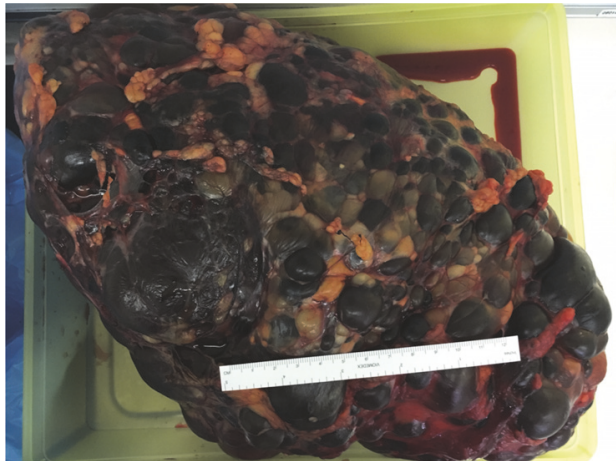

(b)

FIGURE 3: Massive polycystic kidneys leading to an indication for creating space to facilitate transplantation.

TABLE 2: Incidental lesion findings in relation to nephrectomy indication and timing from transplantation.

\begin{tabular}{|c|c|c|c|c|}
\hline Histology & Indication & $\begin{array}{c}\text { Transplant } \\
\text { pre-nephrectomy }\end{array}$ & $\begin{array}{c}\text { Time from Transplant } \\
\text { (months) }\end{array}$ & Follow-up (months) \\
\hline $\begin{array}{l}4 \mathrm{~mm} \text { papillary } \\
\text { adenocarcinoma }\end{array}$ & Pain & Yes & 156 & 17.7 \\
\hline $1.5 \mathrm{~mm}$ papillary adenoma & Space & Yes & 19 & 13.8 \\
\hline $1 \mathrm{~mm}$ papillary adenoma & Space & No & 1 & 6.7 \\
\hline $1 \mathrm{~mm}$ papillary adenoma & $\begin{array}{l}\text { Space, infection, } \\
\text { haematuria, cystic } \\
\text { content change }\end{array}$ & No & l & 2.5 \\
\hline $\begin{array}{l}0.5 \mathrm{~mm} \text { papillary } \\
\text { adenoma }\end{array}$ & Space, infection & No & l & 26.7 \\
\hline
\end{tabular}

who underwent transplantation 5 months after the native bilateral nephrectomy.

An association with polycystic liver disease was found in 18 cases, $\mathrm{p}<0.001$ (independent $\mathrm{t}$-test). Histopathological examination revealed incidental renal neoplasms in five cases (15\%): 1 pTla papillary renal cell carcinoma type 1 measuring $4 \mathrm{~mm}$ and 4 papillary adenomas. Table 2 represents incidental pathological findings in correlation to nephrectomy indication and timing of transplantation. The patient with carcinoma did not require further oncological treatment and is well with no evidence of disease recurrence at 18 months of follow-up. None of those neoplasms were evident in prenephrectomy CTs.

\section{Discussion}

ADPKD patients may get massively enlarged kidneys with resultant problems, where surgery is offered to those approaching ESRD or already in renal replacement therapy. Native bilateral nephrectomy is indicated for patients with large kidneys causing pressure symptoms, pain, infection, bleeding, hypertension, and suspicion of malignancy; this operation is also indicated to create space for a renal allograft [21]. The patients who were transplanted prior to the native bilateral nephrectomy did not experience eGFR worsening postoperatively. The comparison of the preoperative e-GFR was made with the one in the immediate postoperative period since this was the timing of the anticipated maximum effect of the stress of the operation and acute kidney graft injury. There were neither episodes of rejection nor elevations of DSA level for all 26 transplanted patients within the followup period, indicating that there is no immunological risk related to the native bilateral nephrectomy and this was despite the fact that 14 out of the 33 patients required blood transfusion in the postoperative period. These findings also align with previous studies that recommended nephrectomy for polycystic kidneys only after the transplant [22].

In our cohort, the main indication to native bilateral nephrectomy was space $(59 \%)$ in the view of a prospective transplant, or because of compression/abdominal fullness. Recurrent cyst infection was also common (36\%); particular attention has to be given to frail dialysis patients who could develop sepsis more frequently than the general population [23], or even more to the immunosuppressed transplanted patients. In this latter group, there is also a general alert towards the development of malignancy after transplantation, now becoming the leading cause of death with a functioning graft, due to the effect of immunosuppression [24]. In addition to the immunosuppression risk, the role of ADPKD as a risk factor for renal cell carcinoma is well established; in fact a recent review by Yu et al. highlights the association of polycystic kidney disease with the risk of liver, colon, and kidney cancer [25]. Our centre policy is to monitor carefully any cystic change at imaging in ADPKD transplanted patients; 
of note, there is no evidence that there is an advantage in preventing malignancy development in APKD by avoiding the use of calcineurin inhibitors, as immunosuppression alone cannot be accounted as responsible for renal cancer in transplanted patients $[21,26]$.

In the present series, we found five incidental tumours (15\%), of which one was a papillary renal cell carcinoma and the other four were benign adenomas. None of those neoplasms were evident in prenephrectomy CTs. The concept of papillary adenoma is controversial and in theory related to a proliferation in tubulopapillary epithelium that has no metastatic potential. Nevertheless, they are very similar histomorphologically to papillary RCCs [27]. It has been suggested that as the size of papillary adenomas increases so does the amount of chromosomal alteration and the potential to transform to cancer. This raises the possibility that papillary adenomas and papillary RCCs represent a continuum of the same process, as it happens in the pathogenesis of colorectal cancer. Unfortunately, an imaging classification of ADPKD patients to identify those at risk of rapid disease progression or malignancy transformation is not yet standardized; this may be very useful in selecting patients for clinical management.

As reported previously, nephrectomy for enlarged polycystic kidneys in patients with ADPKD is associated with significant complication rates [28]. The most common in our series was the necessity for transfusion, as confirmed by the haemoglobin drop postoperatively in both the transplant and dialysis groups. Literature confirms that avoiding simultaneous nephrectomy and transplantation reduces by approximately $40 \%$ the risk for postoperative complications and by $100 \%$ the risk for blood transfusion [29]. We therefore aim to operate on the patients awaiting kidney transplantation once they are established on dialysis. The only change in anti-HLA level post-native bilateral nephrectomy occurred in a female dialysis patient, with previous history of pregnancies but without being transfused. Eight patients were transplanted after bilateral native nephrectomy without complications.

A death incidence of up to $8.6 \%$ following nephrectomies of APKKD has been reported in literature [30]. There was one death in our cohort ( $3 \%$ ) for a patient who was initially discharged home but died six months later. This patient experienced refractory ascites eventually leading to hepatorenal syndrome (Clavien grade IV). This unfortunate outcome was not predictable on the basis of the liver function tests that were normal preoperatively. Patients with liver involvement require a combined liver-kidney transplantation only in cases of symptomatic hepatomegaly or recurrent cholangitis and if the glomerular filtration rate is $\leq 30 \mathrm{ml} / \mathrm{min} / 1.73 \mathrm{~m}^{2}$ [21]. There is however significant concern in this population for the avoidance of poor postoperative outcomes [31] and there is no standardised practice for their treatment. An association with polycystic liver disease was present in 18 cases in our series $(\mathrm{p}<0.001)$, and the only patient who developed hepatic insufficiency had normal liver function tests, no previous history of liver symptoms, and also a working transplant at the time of the native bilateral nephrectomy and so theoretically was in a lower risk group. The other complications that occurred, i.e., ileus (Clavien grade II) or abdominal collection
(Clavien grade II/III), did not significantly affect the LOS, and 32 of the 33 patients were well in the post-native bilateral nephrectomy follow-up.

\section{Conclusion}

In our experience, native bilateral nephrectomy for ADPKD can be performed safely and it is recommended for patients who need space for a future kidney transplant or have significant refractory symptoms or the suspicion of cancer. Furthermore, although this operation is associated with an increased risk of postoperative transfusion, it does not affect kidney graft function and does not pose an increased immunological risk. We recommend annual screening for the complications that may occur in this particular cohort of patients.

\section{Abbreviations \\ DSA: Donor Specific Antibody \\ Hb: Haemoglobin \\ HLA: Human Leucocyte Antigen \\ LOS: Length of Hospital Stay \\ RCC: Renal Cell Carcinoma.}

\section{Data Availability}

The data used to support the findings of this study are included within the article.

\section{Disclosure}

The preliminary results of this study were presented at the Associations of Surgeons of Great Britain and Ireland annual meeting in held Glasgow, UK, from the 3rd to the 5th of May 2017 [32].

\section{Conflicts of Interest}

The authors declare no conflicts of interest.

\section{Authors' Contributions}

Maria Irene Bellini and Paul Brookes participated in research design, performance of the work, data analysis, and writing the article. Sotiris Charalmpidis collected the data and participated in performance of the work. Peter Hill and Frank Dor participated in performance of the work. Vassilios Papalois participated in performance of work, research design, data analysis, and writing the article.

\section{References}

[1] E. C.-L. Gall, M.-P. Audrézet, J.-M. Chen et al., “Type of PKD1 mutation influences renal outcome in ADPKD," Journal of the American Society of Nephrology, vol. 24, no. 6, pp. 1006-1013, 2013. 
[2] T. Mochizuki, G. Wu, T. Hayashi et al., "PKD2, a gene for polycystic kidney disease that encodes an integral membrane protein," Science, vol. 272, no. 5266, pp. 1339-1342, 1996.

[3] V. E. Torres, P. C. Harris, and Y. Pirson, "Autosomal dominant polycystic kidney disease," The Lancet, vol. 369, no. 9569, pp. 1287-1301, 2007.

[4] S. Klahr, A. S. Levey, G. J. Beck et al., "The effects of dietary protein restriction and blood-pressure control on the progression of chronic renal disease," The New England Journal of Medicine, vol. 330, no. 13, pp. 877-884, 1994.

[5] A. B. Chapman, O. Devuyst, K.-U. Eckardt et al., "Autosomaldominant polycystic kidney disease (ADPKD): Executive summary from a Kidney Disease: Improving Global Outcomes (KDIGO) Controversies Conference," Kidney International, vol. 88, no. 1, pp. 17-27, 2015.

[6] M. A. Lantinga, J. P. H. Drenth, and T. J. G. Gevers, "Diagnostic criteria in renal and hepatic cyst infection," Nephrology Dialysis Transplantation, vol. 30, no. 5, pp. 744-751, 2015.

[7] J. J. Grantham, "Autosomal dominant polycystic kidney disease," The New England Journal of Medicine, vol. 359, no. 14, pp. 1477-1485, 2008.

[8] R. L. Luciano and N. K. Dahl, "Extra-renal manifestations of autosomal dominant polycystic kidney disease (ADPKD): Considerations for routine screening and management," Nephrology Dialysis Transplantation, vol. 29, no. 2, pp. 247-254, 2014.

[9] G. Smedile, M. I. Bellini, G. Iaria et al., "Emergency endovascular repair in a patient with abdominal aortic aneurysm with pelvic transplant kidneys: Case report," Experimental and Clinical Transplantation, vol. 10, no. 6, pp. 601-604, 2012.

[10] A. Persu, M. Duyme, Y. Pirson et al., "Comparison between siblings and twins supports a role for modifier genes in ADPKD," Kidney International, vol. 66, no. 6, pp. 2132-2136, 2004.

[11] A. C. M. Ong, O. Devuyst, B. Knebelmann, and G. Walz, "Autosomal dominant polycystic kidney disease: The changing face of clinical management," The Lancet, vol. 385, no. 9981, pp. 1993-2002, 2015.

[12] R. J. Simms, "Autosomal dominant polycystic kidney disease," $B M J$, vol. 352, article i679, 2016.

[13] T. F. Fuller, T. V. Brennan, S. Feng, S. M. Kang, P. G. Stock, and C. E. Freise, "End stage polycystic kidney disease: indications and timing of native nephrectomy relative to kidney transplantation," The Journal of Urology, vol. 174, no. 6, pp. 2284-2288, 2005.

[14] D. Cohen, M.-O. Timsit, Y. Chrétien et al., "Place of nephrectomy in patients with autosomal dominant polycystic kidney disease waiting for renal transplantation," Progrès en Urologie, vol. 18, no. 10, pp. 642-649, 2008.

[15] T. Sulikowski, K. Tejchman, Z. Zietek et al., "Experience with autosomal dominant polycystic kidney disease in patients before and after renal transplantation: a 7-year observation," Transplantation Proceedings, vol. 41, no. 1, pp. 177-180, 2009.

[16] P. Patel, C. Horsfield, F. Compton, J. Taylor, G. Koffman, and J. Olsburgh, "Native nephrectomy in transplant patients with autosomal dominant polycystic kidney disease," Annals of the Royal College of Surgeons of England, vol. 93, no. 5, pp. 391-397, 2011.

[17] J. B. Wetmore, J. P. Calvet, A. S. L. Yu et al., "Polycystic kidney disease and cancer after renal transplantation," Journal of the American Society of Nephrology, vol. 25, no. 10, pp. 2335-2341, 2014.
[18] A. S. Levey, J. P. Bosch, J. B. Lewis, T. Greene, N. Rogers, and D. Roth, "A more accurate method to estimate glomerular filtration rate from serum creatinine: a new prediction equation. Modification of diet in renal disease study group," Annals of Internal Medicine, vol. 130, no. 6, pp. 461-470, 1999.

[19] D. Dindo, N. Demartines, and P. Clavien, "Classification of surgical complications: a new proposal with evaluation in a cohort of 6336 patients and results of a survey," Annals of Surgery, vol. 240, no. 2, pp. 205-213, 2004.

[20] R. A. Agha, M. R. Borrelli, M. Vella-Baldacchino, R. Thavayogan, and D. P. Orgill, “The STROCSS statement: strengthening the reporting of cohort studies in surgery," International Journal of Surgery, vol. 5, pp. 15-17, 2017.

[21] N. Kanaan, O. Devuyst, and Y. Pirson, "Renal transplantation in autosomal dominant polycystic kidney disease," Nature Reviews Nephrology, vol. 10, no. 8, pp. 455-465, 2014.

[22] H. Ho-Hsieh, A. C. Novick, D. Steinmuller, S. B. Streem, C. Buszta, and M. Goormastic, "Renal transplantation for endstage polycystic kidney disease," Urology, vol. 30, no. 4, pp. 322326, 1987.

[23] K. Doi, A. Leelahavanichkul, X. Hu et al., "Pre-existing renal disease promotes sepsis-induced acute kidney injury and worsens outcome," Kidney International, vol. 74, no. 8, pp. 1017-1025, 2008.

[24] S. A. Acuna, "Etiology of increased cancer incidence after solid organ transplantation," Transplantation Reviews, vol. 32, no. 4, pp. 218-224, 2018.

[25] T.-M. Yu, Y.-W. Chuang, M.-C. Yu et al., "Risk of cancer in patients with polycystic kidney disease: a propensity-score matched analysis of a nationwide, population-based cohort study," The Lancet Oncology, vol. 17, no. 10, pp. 1419-1425, 2016.

[26] M. I. Bellini, J. P. Gopal, P. Hill, D. Nicol, and N. Gibbons, "Urothelial carcinoma arising from the transplanted kidney: A single-center experience and literature review," Clinical Transplantation, p. e13559, 2019.

[27] K. L. Wang, D. M. Weinrach, C. Luan et al., "Renal papillary adenoma-a putative precursor of papillary renal cell carcinoma," Human Pathology, vol. 38, no. 2, pp. 239-246, 2007.

[28] A. H. Bennett, W. Stewart, and J. M. Lazarus, "Bilateral nephrectomy in patients with polycystic renal disease," Surgery, Gynecology and Obstetrics, vol. 137, no. 5, pp. 819-820, 1973.

[29] R. A. Jean, M. Alexandre, and P. S. Yoo, "Kidney transplantation with and without native nephrectomy for polycystic kidney disease: results of the national inpatient sample and the rationale for a 2-staged procedure," Journal of the American College of Surgeons, vol. 226, no. 6, pp. 1079-1084, 2018.

[30] M. A. Kirkman, D. van Dellen, S. Mehra et al., "Native nephrectomy for autosomal dominant polycystic kidney disease: before or after kidney transplantation?" BJU International, vol. 108, no. 4, pp. 590-594, 2011.

[31] M. Veroux, D. Zerbo, G. Basile et al., "Simultaneous native nephrectomy and kidney transplantation in patients with autosomal dominant polycystic kidney disease," PLOS ONE, vol. 11, no. 6, Article ID e0155481, 2016.

[32] M. Bellini, F. Dor, J. Campbell, P. Hill, and V. Papalois, "Polycystic kidney disease and incidental renal neoplasm: one centre experience," British Journal of Surgery, vol. 104, p. 76, 2017. 


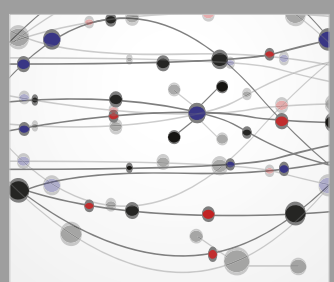

The Scientific World Journal
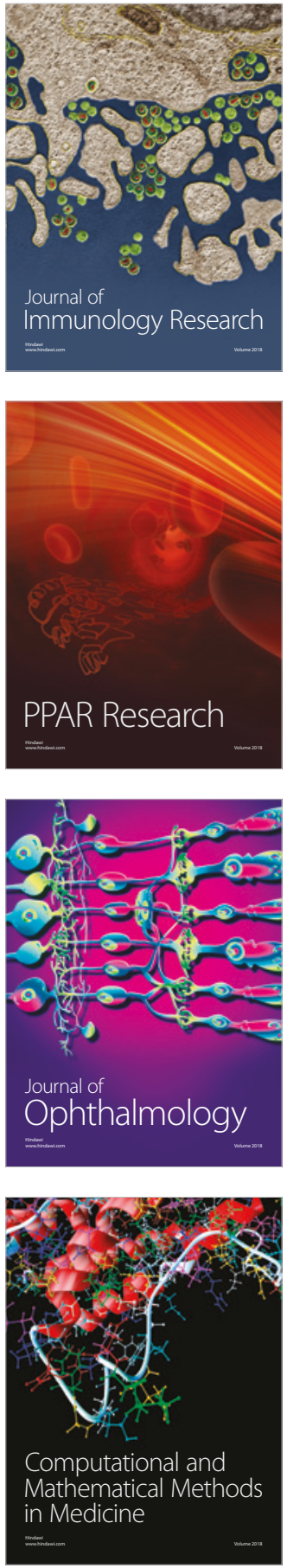

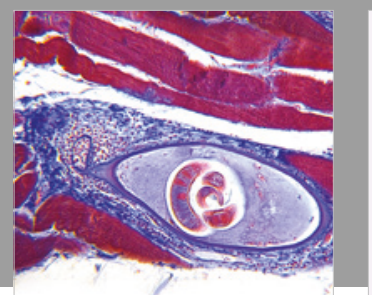

Gastroenterology Research and Practice

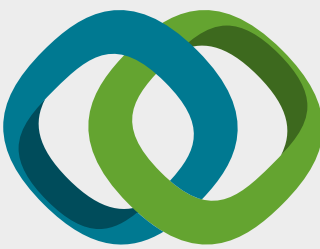

\section{Hindawi}

Submit your manuscripts at

www.hindawi.com
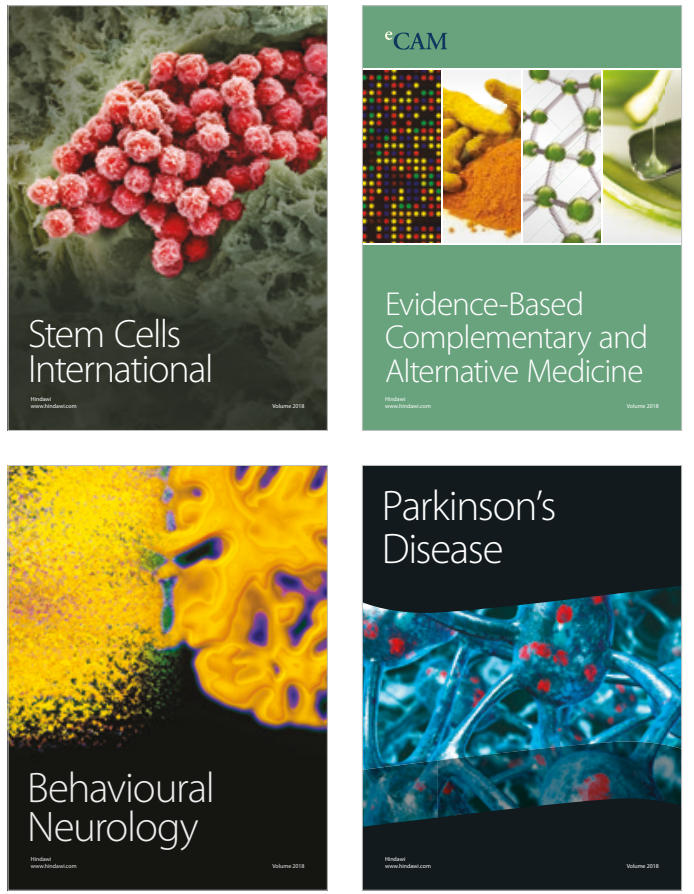

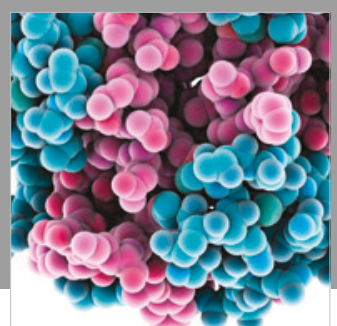

ournal of

Diabetes Research

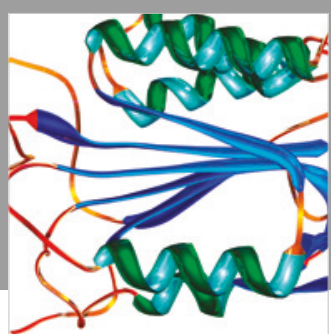

Disease Markers
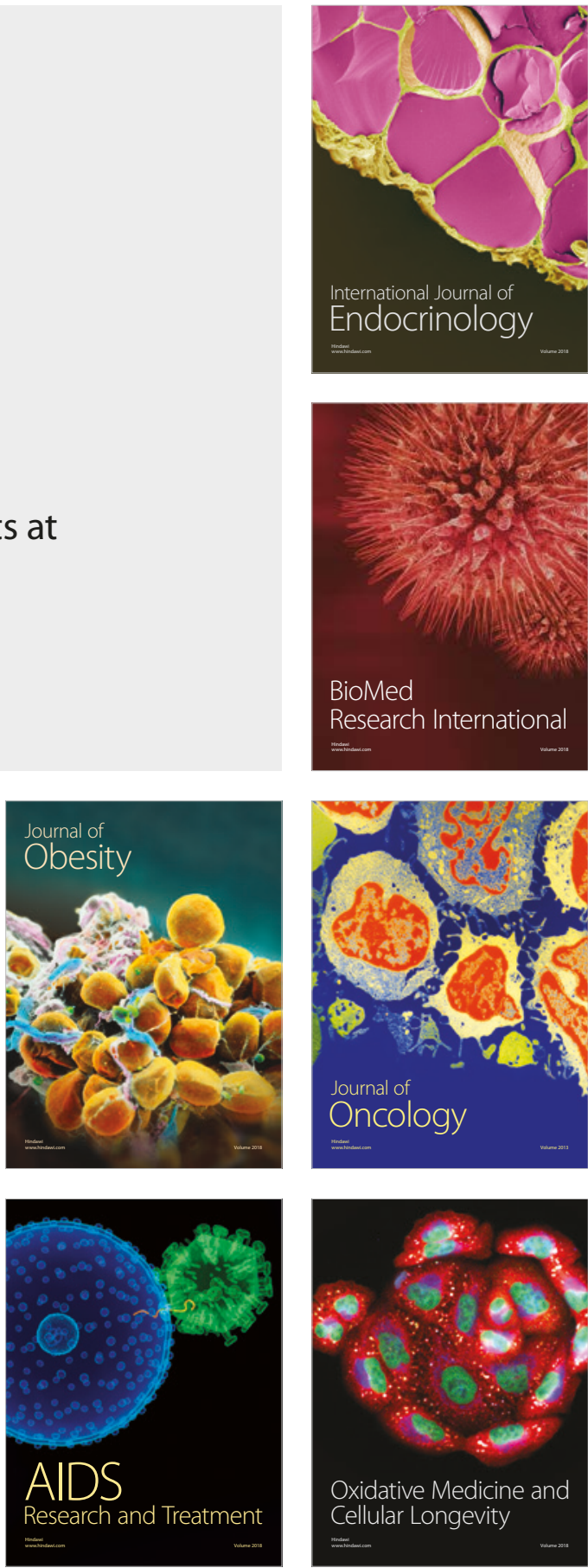